Interactive comment on "The SPACE 1.0 model: A

Landlab component for 2-D calculation of

sediment transport, bedrock erosion, and

landscape evolution" by Charles M. Shobe et al.

Charles M. Shobe et al.

charles.shobe@colorado.edu

Received and published: 18 October 2017

Response to review by F. Clubb

Author's note: reviewer comments are reproduced here, and our responses are italicized.

General comments 
This paper derives a new formulation for modelling of channel evolution incorporating simultaneous erosion of bedrock and sediment transport, set within the Landlab modelling framework. This modelling framework has the potential to be useful to many within the earth science community by tackling a gap between the two end member scenarios of detachment- and transport- limited models: although previous models between these have been proposed, the inclusion of the authors' model within the Landlab framework allows it to be used easily by the community and for the results of the authors' study to be reproduced, as well as being applicable over large spatial and temporal scales. The manuscript is well written, clear, and the derivation of the model is well laid out. I therefore believe that the paper is suited to publication within GMD following to some corrections which I have specified below.

We thank the reviewer for taking the time to review this manuscript, and for their helpful suggestions. We have endeavored to thoroughly address all comments below.

\section{Specific comments}

Abstract: previous models have been presented in the literature that include combine erosion and deposition, which the authors review in Section 2, but there is no mention in the abstract of the novelty of the authors' approach compared to these previous methods. The abstract should describe precisely why a new modelling approach is needed for this problem.

In our revisions we have tried to stress that the novelty of our approach involves the ability to transition between transport- and detachment-limited states (which some existing models can do), the ability to erode sediment and bedrock at the same time (which some existing models can do), but most importantly the ability to do both of those in a

Printer-friendly version

Discussion paper

\section{(1)}


2-D landscape evolution model that is designed to be coupled to other models and run over landscape evolution space and time scales. Basically, we feel that we are incorporating the best features of existing models (with a slightly different mathematical formulation) into an extensible and efficient landscape evolution modeling tool. Further, we are expanding upon existing models by applying the explicit erosion/deposition framework to a mixed bedrock-alluvial channel model. We have changed the abstract to add: "Modeling landscape evolution over large spatial and temporal scales requires a model that can 1) transition freely between transport-limited and detachment-limited behavior, 2) simultaneously treat sediment transport and bedrock erosion, and 3) run in 2-D over large grids and be coupled with other surface process models.", and "SPACE improves on previous models of bedrock-alluvial rivers by explicitly calculating sediment erosion and deposition rather than relying on a flux divergence (Exner) approach."

Page 1, Lines 18 - 20: sentence needs reworded.

We broke the first sentence up into two to improve readability and clarity. It now reads: "Rivers are the primary agents of land-surface lowering in non-glaciated landscapes (e.g., Whipple, 2004). Erosion and sediment transport in rivers affect human river management (e.g., Graf et al., 2010), landscape mass balance (e.g., Armitage et al., 2011), and global biogeochemical cycling (e.g., Hilton, 2017)."

Page 2, Line 5: '...the superiority of one model over the other in tests against real landscapes...: I think it would be useful to include in the introduction some examples of how these different models have been tested against real landscapes. A brief review of this would be useful to the reader, and set the context for providing some demonstration of the potential of using equivalent tests for the SPACE model.

Printer-friendly version

Discussion paper 
We have written a new section (section 2) to discuss how these models have been tested against one another. This, as noted by the reviewer, will help provide context for our later discussion of how SPACE could be tested in the field (section 9).

Page 4, Lines 1 - 7: The authors could expand here upon what the limitations are of the erosion-deposition models of Lague (2010) and Zhang et al. (2015).

We have re-written the review to more fully educate readers about the pros and cons of the many different modeling approaches out there. This includes a statement about the advantages of the SPACE algorithm over those two models, which are that 1) SPACE uses explicit calculation of sediment erosion and deposition instead of an Exner-style conservation rule, and 2) SPACE is easily applied to landscape evolution modeling exercises because of its status as a Landlab component.

Page 4, Lines 15-17: Other models have been developed that model mixed bedrock alluvial channels that are not mentioned here, as noted by Reviewer 1. I think it is worth mentioning here the difference between the reach-scale approach generally taken by the development of these mixed bedrock-alluvial models compared to the whole landscape scale that is used as a framework in this paper, to demonstrate the need for and motivation behind development of the SPACE model. It would also be good to discuss the challenges behind scaling reach-scale models up to whole landscapes.

As we note in our response to reviewer 1, we have extensively re-written and expanded our literature review (section 3) to take these other contributions into account. The new review contains a discussion of several of these models that are focused on reachscale cover dynamics. At that point, we discuss why some of these models are likely not to scale well for landscape evolution applications (the computational cost of solving

Printer-friendly version

Discussion paper 
2-D flow equations, for example). We hope that this shows the reader why SPACE is an important contribution.

Page 5, Eq 1: Do you think that including a variable channel width would be possible in the model, or would this be too computationally expensive to do for large spatial/temporal scales? I know this is discussed in the previous section as processes which are not treated in the model, but it would be interesting to have an idea of which processes would potentially be possible to include in future developments, and what isn't due to scale issues.

This is a good question. As numerous authors have pointed out, the "default" channel width closure (i.e., width goes as the square root of discharge) is computationally efficient but not physically-based. It would indeed be possible to incorporate dynamic channel width into the model. We view the approach of Lague (2010), in which stresses are partitioned between the channel bed and banks according to a trapezoidal crosssection approximation, as one potential way of incorporating width changes. There are also more complex approaches for calculating or approximating the shear stress across the channel cross-section, which we discuss in the added text below. We have not attempted to incorporate such rules into the SPACE model, and so cannot be sure what the computational cost would be, nor how that cost would scale with grid size. Our rationale for excluding dynamic width from the SPACE model is both potential computational cost but also the substantial number of parameters already necessary to describe the coupled evolution of sediment and bedrock. Adding an empirical or semi-empirical width adjustment rule would substantially increase model complexity.

In order to make it clear to the reader that dynamic width could be incorporated, we have added the following in section 4.3:

"While we employ a simple closure for channel width in which width scales as the

Printer-friendly version

Discussion paper
Interactive

comment 
square root of water discharge (Leopold and Maddock, 1953; Wohl and David, 2008), it may be desirable for some applications to add dynamic channel width adjustments to the model, as previous work has suggested that width trades off with slope in transient channels (e.g., Finnegan et al., 2005; Turowski et al., 2006; 2009; Wobus et al., 2006; Whittaker et al., 2007; Attal et al., 2008; Lague, 2010; Yanites and Tucker, 2010). One option for incorporating dynamic width is to calculate or approximate shear-stress distributions across channel cross-sections (Kean and Smith, 2004; Wobus et al., 2006; 2008; Turowski et al., 2009). A simpler dynamic width rule can be obtained by partitioning erosive power between the bed and banks under a trapzoidal channel assumption (Flintham and Carling, 1988) as detailed in (Lague, 2010). Different approaches have different numbers of parameters and computational costs, and further work will be necessary to elucidate which advances beyond the standard empirical width closure are tractable within the SPACE landscape evolution model framework."

Figure 2: It seems like it would be possible to include the tool effect as well as the cover effect, where $E_{r}$ would decrease where $H / H_{*}=0$ as there are no tools for effective incision into bedrock? Is there potential for this to be included as an option in the model formulation?

It is indeed possible to include the tools effect, but we suggest that a more effective way to do so would be to assert that $E_{r}$ increases with $Q_{s}$ until $Q_{s}$ reaches transport capacity (where transport capacity is $Q_{s}$ when erosion equals deposition, or $E_{r}+E_{s}=$ $D_{s}$ ). The advantage of this approach is that bedrock erosion actively responds to the amount of sediment in transport, not the amount of sediment resting on the bed. The cover effect, or reduction in $E_{r}$ as $H / H_{*}$ increases, would compete with the increase in $E_{r}$ as $Q_{s}$ increases. We do not incorporate this effect into the current version of SPACE because it complicates model comparison with analytical solutions, but it could certainly be incorporated into future versions.

Printer-friendly version

Discussion paper 
We agree with the reviewer that it would be helpful to explain to the reader how the tools effect could be incorporated. To that end, we have added two sentences to the end of section 3.3: "The "tools effect" on bedrock erosion could be incorporated into the model by assuming that $E_{r}$ at a given $H / H_{*}$ increases with $Q_{s}$ until $Q_{s}$ reaches transport capacity (which is $Q_{s}$ when $E_{s}+E_{r}=D_{s}$ ). Because increases in $H / H_{*}$ already account for the "cover effect," the $E_{r}$ dependence on $Q_{s}$ need only be positive and not decline with increasing $Q_{s}$ (Sklar and Dietrich, 2001; 2004; Gasparini et al., 2006; Turowski et al., 2007)."

Page 8, Line 33: It would be useful to explain 'state function' in some more detail here.

In response to this comment, the following comment, and comments from the other reviewer, we have re-structured and re-worded section 4.3.1 (see response to next comment below). In doing so, we no longer use the term 'state function.'

Page 9, Line 6: What is the justification for choosing an exponential decline in erosion at the threshold value here?

The idea here is to account for the fact that sediment entrainment and bedrock erosion thresholds in nature cannot generally be characterized by a single value. Rather, the threshold for a given population of sediment grains or a given areal exposure of bedrock is likely to be a distribution of values. The distribution will be influenced by such variables as grain size and sorting, flow history, and sediment flux for sediment, and weathering, local mineralogy, and joint spacing and orientation for bedrock (see our revised section 4.3.1). We chose an exponential function because it accounts for a smoothed threshold while still yielding the behavior expected from a single-value threshold. For example, when stream power is far above the user-defined threshold, excess stream

Printer-friendly version

Discussion paper 
power is $\omega-\omega_{c}$ just as in single-threshold cases. Conversely, when stream power is far below the defined threshold, the threshold term approximately equals $\omega$ (the available stream power), meaning that entrainment or erosion is effectively zero. The other benefit of choosing an exponential function over some other form is that it does not require any additional model parameters because the user-defined threshold scales the exponential function.

To make all of this clear to the reader, we have re-written section 4.3.1. We focus on summarizing support for the idea of a distribution of thresholds rather than a single number, and we have added a sentence explaining that we prefer the exponential because it adds no model parameters: "We chose an exponential function because it allows for smoothing of entrainment and erosion thresholds, and therefore honors the reality that such thresholds tend to be distributions of values rather than a single value, without adding any model parameters."

Page 12, Line 27: Is there a specific value of $\mathrm{q} / \mathrm{V}$ above which the model predicts detachment-limited behaviour? It would be good to clarify this here.

In the erosion/deposition model presented by Davy and Lague (2009), there is a dimensionless number $\frac{V}{r}$ that predicts the behavior. When $\frac{V}{r}>1$, behavior is transportlimited and vice versa. However, this only holds when eroding a single layer, as incorporating multiple layers increases the number of factors that influence sediment entrainment (for example, in SPACE, $H / H_{*}$ has a strong influence). There is not a specific value that governs the DL/TL transition in the SPACE model, because the dimensionless ratios presented in this section do not incorporate all of these effects. Still, we think that it is worth presenting the simple case from Davy and Lague (2009), so we have added text to say:

"Specifically, following Davy and Lague (2009), we can define a dimensionless number

Printer-friendly version

Discussion paper 
$\frac{V}{r}$ that governs the transition between detachment-limited and transport-limited dynamics. In the sediment-only case (when $H \gg H_{*}$ ), or in the bedrock-only case $(H=0)$, $\frac{V}{r}>1$ gives transport-limited behavior and $\frac{V}{r}<1$ results in detachment-limited behavior (Davy and Lague, 2009). In cases where sediment and bedrock are eroded simultaneously, especially if there is a significant erodibility contrast between the two, the behavior is not so easily predicted and will generally contain contributions from both detachment and transport limitations."

Page 13, Line 9: add in reference to equation 11 here.

We have added the reference.

Page 13, Equation 29: I like the within-cell calculation of sediment flux!

Thank you!

Page 14, Line 1: If $Q_{s}=Q_{s}^{\text {out }}$, does this imply that all of the deposition happens at the downstream node of each model cell?

Good question. The local analytical solution for $Q_{s}$ was designed to account for the fact that 1) rapid sediment entrainment should decline over a cell length as it depletes the available sediment, and 2) rapid deposition should decline over a cell length as $Q_{s}$ gets smaller. Using $Q_{s}=Q_{s}^{\text {out }}$ allows us to achieve this outcome, whereas using $Q_{s}=Q_{s}^{\text {in }}$ would allow for over-erosion or over-deposition, depending on the value of $Q_{s}^{i n}$.

To answer the reviewer's specific question: using $Q_{s}=Q_{s}^{\text {out }}$ allows us to correctly account for the sediment deposited within a cell, but that sediment is deposited over

Printer-friendly version

Discussion paper 
the whole length of the cell.

In response to this comment as well as one from the other reviewer, we have slightly altered this sentence to clarify why we equate $Q_{s}$ and $Q_{s}^{\text {out }}$ for the deposition term: "Because sediment deposition in a cell depends on both $Q_{s}^{\text {in }}$ from upstream and sediment entrained from the cell itself, we can substitute $Q_{s}^{\text {out }}$ for $Q_{s}$ in the deposition term. Eq. (29) may then be solved to yield the local analytical solution for $Q_{s}$ within a model cell:"

Interactive

Figure 4: It would be useful to have a plot of a run where you vary the ratio of $E_{s}$ to $D_{s}$ to demonstrate how this could affect the sediment thickness as well as the plots where you vary the parameters independently.

We have added two panels to Figure 4 to address this comment. One shows the results of varying $E_{s}$ and $D_{s}$ simultaneously in order to vary the ratio between the two, as requested by the reviewer. The other shows the result of keeping a constant ratio of $E_{s}$ to $D_{s}$ while the magnitudes of the two parameters change. While the reviewer did not ask for this specific addition, we felt like the behavior (that lower values of $E_{s}$ and $D_{s}$, even if their ratio is the same, result in a longer adjustment timescale for $H$ ) was worth pointing out.

Page 18, Line 9: Have you assessed the stability of the model to the timestep? How stable is the model at greater timesteps? 1 year seems like a very short timestep if you wanted to run the model over geological timescales.

The model is generally stable at timesteps greater than one year. The example in the code guide is stable at 10-year timesteps with the default parameterization, but becomes unstable at 50-year timesteps. We used a 1-year timestep in the text and $\mathrm{C} 10$

Printer-friendly version

Discussion paper 
example code 1) to ensure high numerical accuracy for comparison with analytical solutions and 2) to allow readers/users to change parameters with a lower likelihood of making the model unstable. We have heuristically assessed model stability with respect to the timestep over different model parameterizations and grid resolutions, and found that in general, 10-year timesteps are stable in most common modeling scenarios. However, we are unaware of a Courant-Friedrichs-Levy-type criterion that could be formally defined for the numerical implementation of SPACE. The stability of SPACE depends strongly on the strength of the $E_{s}$ and $D_{s}$ terms. For example, when $K_{s}$ and $V$ are high such that sediment is easily entrained and deposited, the model is stable at a smaller timestep than it is when $K_{s}$ and $V$ are low (i.e., when the river is acting in a detachment-limited way).

A simple back-of-the-envelope calculation for a stability criterion is to assume a sediment-mantled bed of a given slope and calculate the time required for an upstream node to erode enough that its slope is lower than its downstream neighbor. For example, consider an end-member case of $H \gg H_{*}$ in which two nodes separated by distance $d x$ lie along a slope of 0.001 such that the vertical drop between them is 0.1 $m$. In this case, neglecting any entrainment threshold and assuming no sediment flux from upstream, $E_{s}=K_{s} q S^{n}$. The maximum timestep that will prevent the upstream node from becoming lower than the downstream node is $0.1 \mathrm{~m}$ divided by $E_{s}$. As such, the maximum stable timestep shrinks as $K_{s}$ and $q$ increase.

In response to the reviewer's question, we have added text to section 6 briefly making the point that the stability of SPACE is subject to its specific parameterization, but also that we have generally found it to be stable for 10-year timesteps. This new text reads: "While the SPACE 1.0 component is stable at 10-year timesteps under most conditions, we use a timestep of 1 year here to maximize numerical accuracy for comparison with analytical solutions."

Section 6: Although testing of the model against the analytical solutions is useful in

Printer-friendly version

Discussion paper 
showing that the numerical implementation is working (and it's really nice to see that it can replicate both detachment-limited and transport-limited behaviour), this does not evaluate the applicability of the method to real landscapes, especially as the analytical solutions are from the same framework as that of SPACE model (e.g. detachmentlimited stream power and transport-limited eqns). What is really interesting is to know how we could validate the model predictions against real landscapes. I think the paper would be improved if the authors could provide some recommendations of how their model can be tested on real landscapes (either natural or experimental), maybe as a section in the discussion (I'm aware it's a model description paper, so actually performing these validations is probably beyond the scope here).

This comment was echoed by the other reviewer as well, and we have added a section to the paper (section 9.1) to address how the model might be validated against real landscapes. As the reviewer notes, we view performing such tests as beyond the scope of this model description paper, but we hope that our discussion will provide avenues for future model validation efforts. In section 9.1 we discuss 1) potential validation of the steady-state predictions that SPACE makes for channel slope and sediment thickness, and 2) potential validation of the transient predictions, including the transient differences in concavity between the sediment surface and the bedrock surface, as discussed in the reviewer's next comment.

Figure 8: This figure is really interesting. I wonder if it would be possible to compare the different concavities of the channel profiles predicted from the bedrock surface and that of the alluvial layer. Does the concavity of the profile through time give some indication of how detachment-limited or transport-limited the model is at that point? I wonder if in real landscapes this could give an indication of transience, or for the transition between detachment- and transport-limited conditions downstream along channels.

Printer-friendly version

Discussion paper 
This is an interesting point and we have endeavored to briefly address it in the new section on comparing SPACE to field data (see response to comment above). One potential pitfall that would need further exploration is the role of discharge variability. When we measure something like sediment thickness (or the surface profile of a sediment layer) in the field, it is unclear whether that particular measurement is representative of the temporal mean, given that the most recent flow events can have a strong control on sediment thickness. To fully explore the benefits/problems of the comparison the reviewer suggests is a topic for another day, but we have added some text to section 9.1 to make readers aware of the potential opportunity:

"In the transient case, the relationship between the longitudinal profile of the bed sediment surface and that of the bedrock may be useful for validating SPACE model predictions. For example, Fig. 8 shows that for an uplifting landscape with zero initial sediment thickness, SPACE predicts bottom-to-top alluviation of the channel profile. In this case, the sediment surface does not reflect the steepened reach commonly associated with the propagation of transient signals up a river profile, while the bedrock beneath does. The prediction of SPACE is therefore that in a transient river profile with some amount of bed sediment, the concavity of the sediment surface is not expected to match that of the bedrock surface. The difference in concavity between the sediment surface and the bedrock surface should then decline as channels approach steady state, a prediction that is testable in a landscape where channels exist in different stages of transient adjustment. It is important to remember that the sediment thickness predicted by SPACE is a spatial average within a model cell. Further, using realistic (i.e., time-varying) flow distributions to force the model would result in temporal variability in sediment thickness (Lague et al., 2005; Lague, 2010), complicating the interpretation of sediment thickness values from a specific field campaign. While testing the steady-state predictions of SPACE is likely feasible in well-constrained landscapes, the transient dynamics may be best explored in a laboratory setting."

Printer-friendly version

Discussion paper

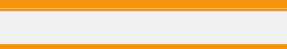

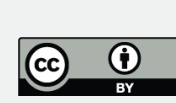


Code availability: I like the iPython notebook, it was really easy to use and gives a good idea of the model structure.

\section{Thank you!}

Interactive comment on Geosci. Model Dev. Discuss., https://doi.org/10.5194/gmd-2017-175, 2017. 